\title{
City Scenario based Traffic Handling and Congestion Avoidance in Vanet
}

\author{
Ravi Yadav \\ Research Scholar, \\ KNIT Sultanpur, \\ India
}

\author{
Awadhesh Kumar \\ Professor, \\ KNIT Sultanpur, \\ India
}

\begin{abstract}
Vanet is a special category of adhoc-network in which vehicles and other stationary unit (RSU) form network for special need or purpose by inter-vehicle communication. Since in recently decades, VANET gaining tremendous attention of research for making reliable communication among vehicles for easy navigation and better handling of traffic .Since due to success of VANET reliability, there is addition of large number of vehicles running on road.As a result large number of vehicles creates congestion(traffic) in city which further result in pollution, speed factor and various other problem for managing traffic .In this paper, we introduced concept of odd-even policy for handling of traffic and smooth transportation and better communication among Vehicles in Vanet .
\end{abstract}

\section{Keywords}

$$
\text { VANET,NS2,ODD-EVEN Policy. }
$$

\section{INTRODUCTION}

An adhoc-network can be defined as collection of nodes forming dynamic network without central administration or infrastructure that usually formed in mobile communication or telecommunication[1]. VANET is type of mobile adhocnetwork where vehicles (nodes) form a network for better safety and comfort among vehicle user. VANET technology integrates new generation wireless network capabilities with vehicles for better navigation .It build robust adhoc -system between moving vehicles and stationary access unit (RSU).VANET uses different wireless network tools such as WIFI $802.11 \mathrm{~b} / \mathrm{g}$,WiMax ,Bluetooth etc.[2] for communication among vehicles and roadside unit.

Vanet mainly focus on providing information related to traffic management, safety and other vital information related to roadway situation. Since this network is infrastructures so each vehicle is equipped with Vanet device(on board unit) for receiving and relaying message in wireless network. To develop reliable ,safe-full, and efficient system for moving vehicle ,there is introduction of Intelligent Transport System(ITS). ITS consists VANET as vital component which includes Vehicle -to-Vehicle Communication(V2V), Vehicle to Roaside-infrastucture(V2R) and Inter-Vehicle communication(IVC)[3].

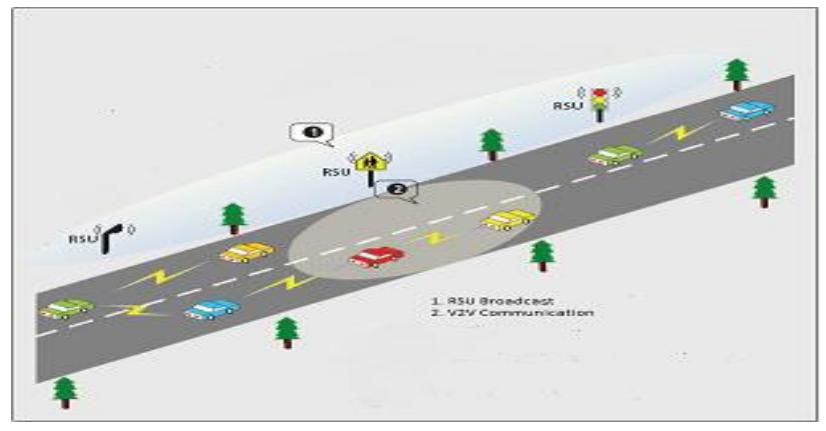

Fig 1. Introduction to VANET

VANET mainly constitute three entities[5] :-

- Roadside Unit:- this is a unit which are stationary in nature and used for monitoring and providing useful information to moving vehicles. Mainly the communication between vehicles and Roadside unit is single hop or multi-hop communication.

- Vehicle :-These are generally nodes in VANET. Vehicle communicates wireless among vehicle and roadside unit for information transmission and gathering.

- Communication Medium: We RF waves for communication among entities in VANET .

Characteristics of VANET

- $\quad$ Self organizing network

- Robust, Flexible and Scalable.

- High Mobility

- Dynamic Network topology

- No Constraint of power

- boundless Network Size

- Variable network Density

- Increased Computation ability.

Application of VANET:-

- Road Traffic Safety

- Traffic Engineering

- Comfortable and Eased Road Travel.

- Pollution Control.

In our paper Section 1 contain Introduction to VANET ,its characteristics and application. Section 2 Contain our traditional System of handling traffic .Section 3 contain our proposed system for efficient handling of traffic. Section 4 contain our result and analysis of our proposed system. Section 5 highlights the proposed system and our future research area. 


\section{EXISTING SYSTEM}

In Traditional Existing System, there is no concept of VANET in real world scenario. Simply all the vehicles on road follow a fixed path without having communication either between vehicle i.e (V2V)or with road side unit(RSU)[4].In our traditional existing it depend upon all the driver capability to overcome and process all the situation for better and eased traveling. In Existing System ,the traffic(congestion) maintained at high-populated area by Traffic-light System. In Traffic-Light Signal System, all the vehicles follow traffic rules based on signal color of light- system. Generally these Traffic -light Signal System are set up at intersection point of two or more roads. The Traffic-light System operates in three signal color modes i.e red, green, yellow. The red signal color indicates that the vehicles has to stop its movement the green signal color indicates that vehicles can start it movement mode while yellow signal color indicates that the speed of vehicle must slow down followed by red color. All the duration of signal color display at traffic light-system is manually carried out by person who monitor traffic. The one with road having more traffic has very less display time for red signal and vice -versa.

Since in our Existing system, all the vehicles follows same route for travel without intercommunicating with each other . If that route contain traffic it further

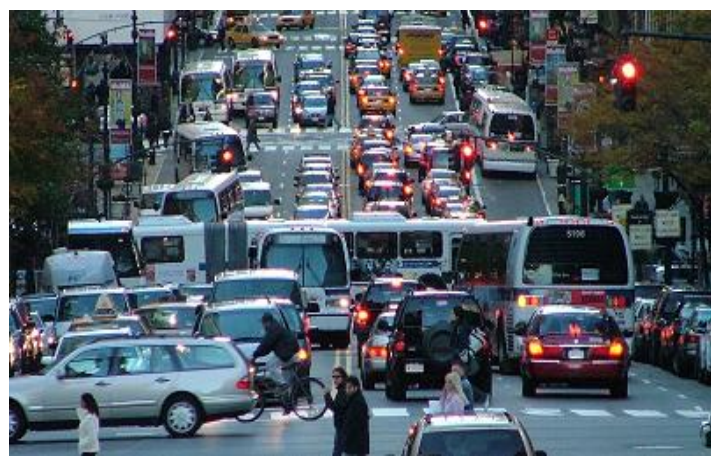

Fig 2. Existing System of Traffic Management

creates more traffic (congestion) on road due to lack of communication.This result in more difficulties in handling of traffic. The problem of our Existing System is that on any high traffic - road if accident occur the medical facilities cannot be fastly provided as no ongoing vehicles is unaware of that situation making worse situation. Also in our Existing System the high density of vehicles is difficult to control and monitor. Due to increase in vehicle density, there is rapid increase in air pollution, noise pollution, fuel consumption and time required to reach from one place to another place[6]. To overcome all this problem we proposed a system based on Odd-even policy in Vanet.

\section{PROPOSED SYSTEM}

In our proposed System we tried to improve traffic handling in Vanet by using odd even policy mechanism[7]. The ODDEVEN mechanism reduces the vehicle density just run on the road on particular date. If it is odd date then only odd number license-plate vehicles are allowed to travel in city and if it is even date then only even number license-plate vehicles are allowed to travel in city[8][9].If on odd date if even-number license plate vehicle is travelling the RSU will just inform by sending reroute message to even number license-plate .Since in every vehicle there is onboard unit equipped which is used to relay communication among RSU as well with other vehicles. Onboard unit is combination of both transmitter as well as receiver.

In our proposed work, at RSU firstly there is computation whether today day is odd day or even day . Once the information is obtained it is stored at RSU. Now if any vehicle comes within ranges of RSU then vehicles sends a request message for finding whether which type of vehicles i.e odd or even number license-plate vehicles are allowed. Now RSU will reply by sending message that only odd

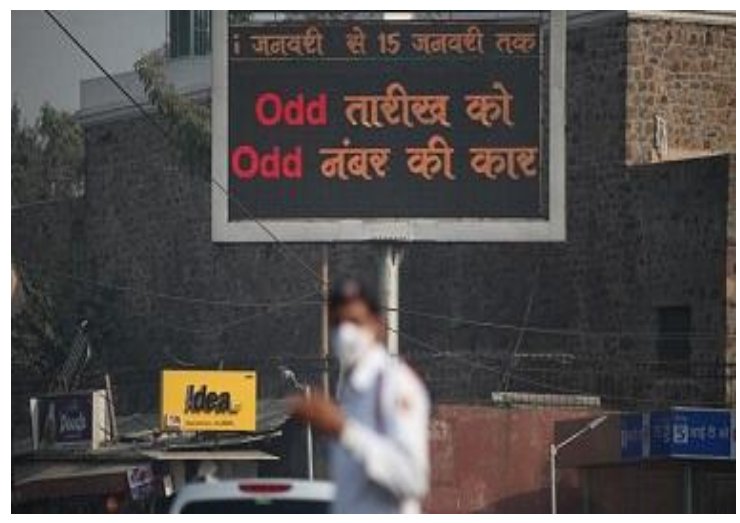

Fig 3. Odd-Even System

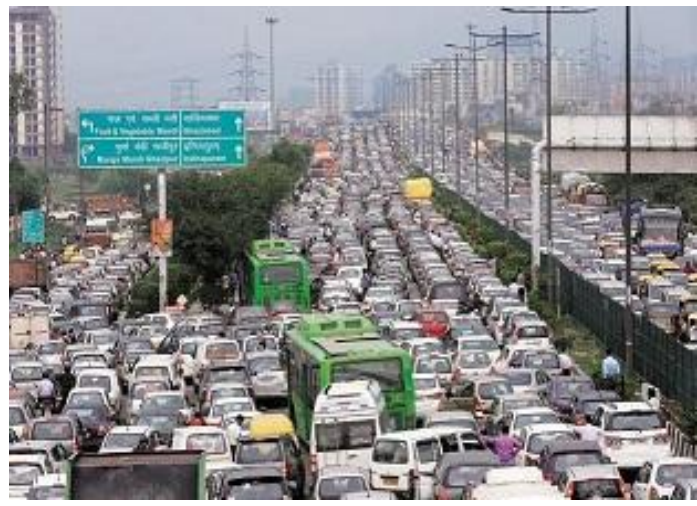

Fig 4. Congested Existing System

number vehicles are allowed if it is odd day and vice-versa to onboard unit equipped in vehicles. Now RSU will broadcast message to all the vehicles approaching towards itself. The vehicles are out of range of RSU will communicate using vehicle-to-vehicle communication. RSU will also monitor all the vehicles that approaches toward himself. If any even number vehicle arrives on odd day then RSU will forward message to that vehicles to reroute its movement back from where it comes.In our proposed system we assume only ordinary vehicles ,i.e.not included VIP Vehicle ,ambulance or Police Vehicle or any other Emergency Vehicle.

Our proposed System can be viewed by the following flow chart. 


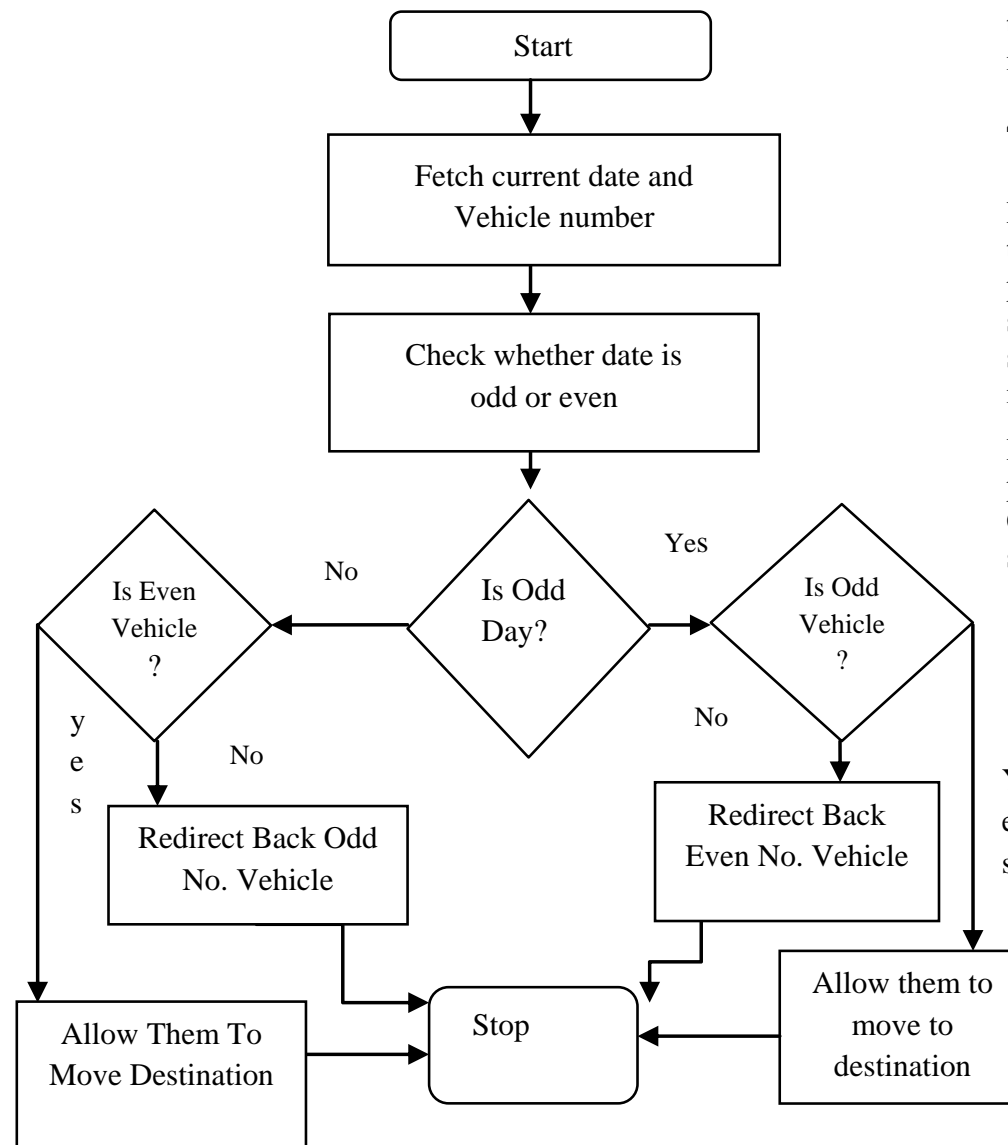

Fig 5 .Flow Chart For Our Proposed System Based on ODD-EVEN Policy

Step 1-. First step is fetch current date from system Clock .In this Step we also Fetch the vehicle number of each vehicle.

Step 2 -Second Step is to check whether our fetch date is odd or even date. If it is divisible by 2 then it is even day else it is odd day

Step 3- If the compute date is odd date, then two condition occur

i. If odd day and odd number vehicle arise ,then allow that vehicle to move to its destination.

ii. Else Even number vehicle is rerouted back to its original position of movement.

Step 4- If computed date is even day ,then two condition arise

i. If on even day even number vehicles arise then allow that vehicles to move to its destination.

ii. Else odd number vehicle are reroute back to its original position of movement

Step 5- Repeat Step 3 and Step 4 untill no vehicle remains to monitor.

In our proposed system as we can easily monitor and control the vehicle density that travelling on particular day, we can also easily control traffic handling and management. Due to decrease vehicle density there is decrease in air pollution, noise pollution etc. In Delhi by using odd-even policy the pollution level decrease by $2 \%$ due to vehicle pollution. Also odd-even policy help in reducing fuel consumption in particular area in month. And the time consume from travelling from one place to another place is reduced as we increase our vehicle speed due to less vehicle density.

\section{SIMULATION RESULT AND ANALYSIS}

During start of simulation, the network simulator initialize the current network by providing position of RSU, vehicle position and all other units position required in our proposed system. The information regarding vehicle and current day is stored in RSU and each vehicle is assign a default number for its identification.

Before beginning of simulation we consider following parameter asshown in previous table for Simulation Configuration.After configuring simulation, we consider some parameters for performance Evaluation.

Table 1. Simulation Parameter

\begin{tabular}{|l|l|}
\hline Parameter & Value \\
\hline Routing Protocol & AODV,DSDV \\
\hline Simulation time & T time(ms) \\
\hline Number of Nodes & N nodes \\
\hline Channel type & Wireless \\
\hline Topography Dimension & N*M sq.m \\
\hline Queue Length & 50 \\
\hline Speed & $50 \mathrm{~m} / \mathrm{s}$ \\
\hline MAC protocol & IEEE 802.11 \\
\hline
\end{tabular}

\subsection{Packet Delievery Ratio:-}

It can be defined as ratio of number of packets received to number of packets sent. More is PDR better is our System.

PDR=Number of packet received /Number of packet sent

\subsection{End To End Delay}

It can be defined as time difference between the packet arrive at destination to time packet sent

Delay $=£$ (arrive time-sent time) $/ £($ number of Connection)

Less is delay value better is simulation

\subsection{Throughput}

It can be defined as amount of data that is delievered from one node to another node in unit time.More is throughput better is our System.

Here we compare our proposed System when we useTwo different routing protocol. 


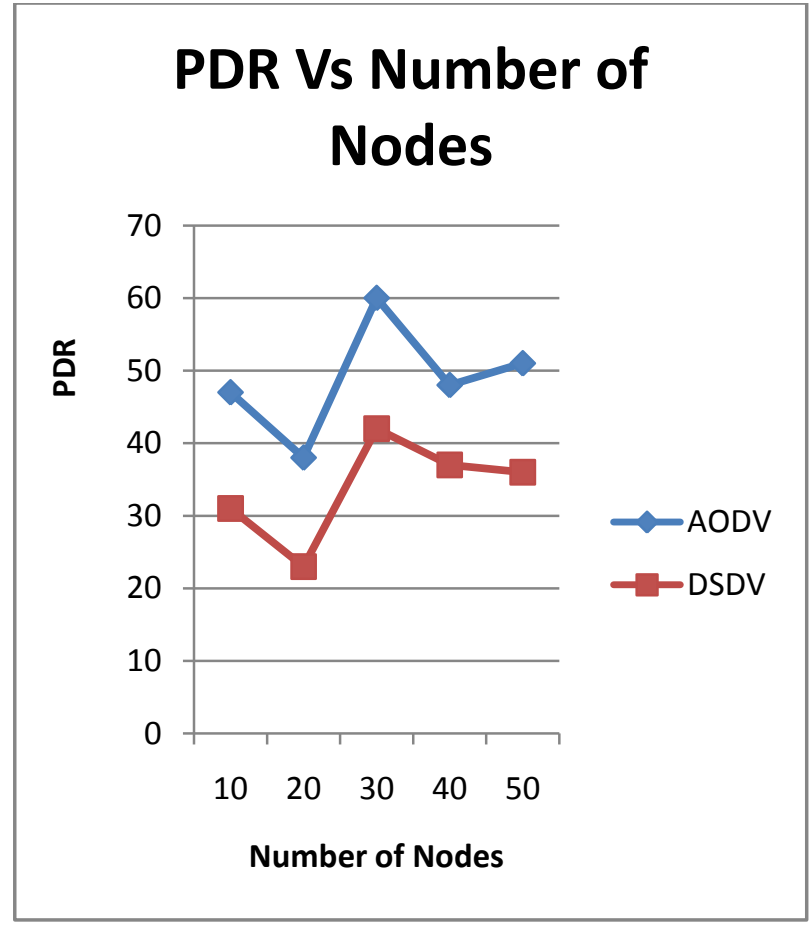

Fig 6. PDR Analysis

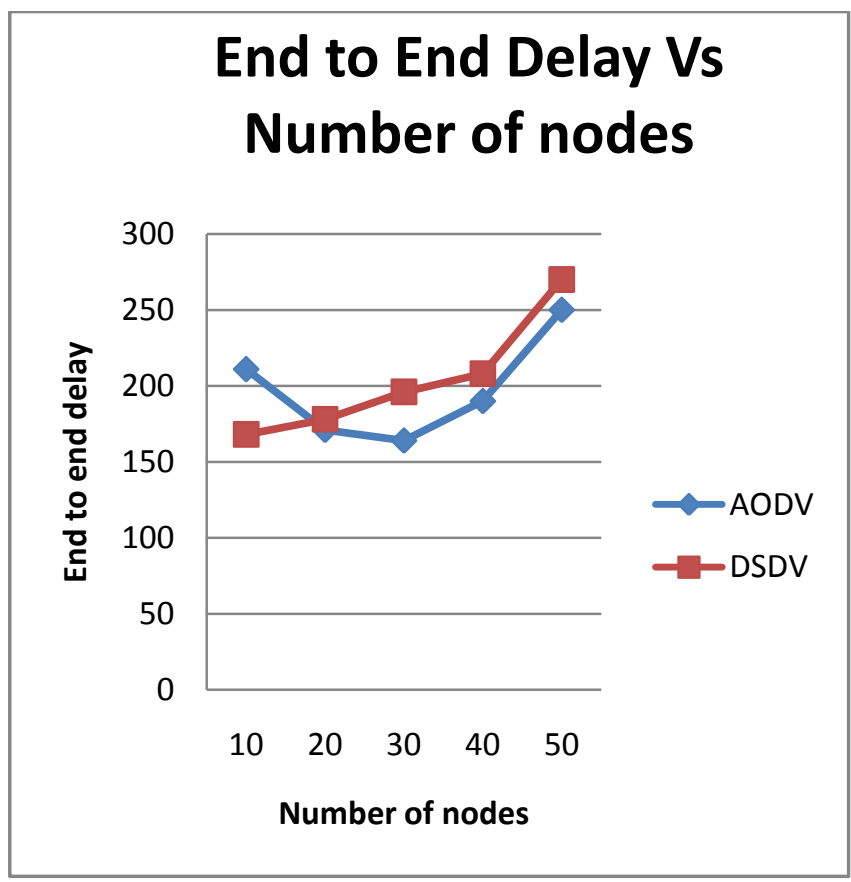

Fig 7. End To End Delay Analysis

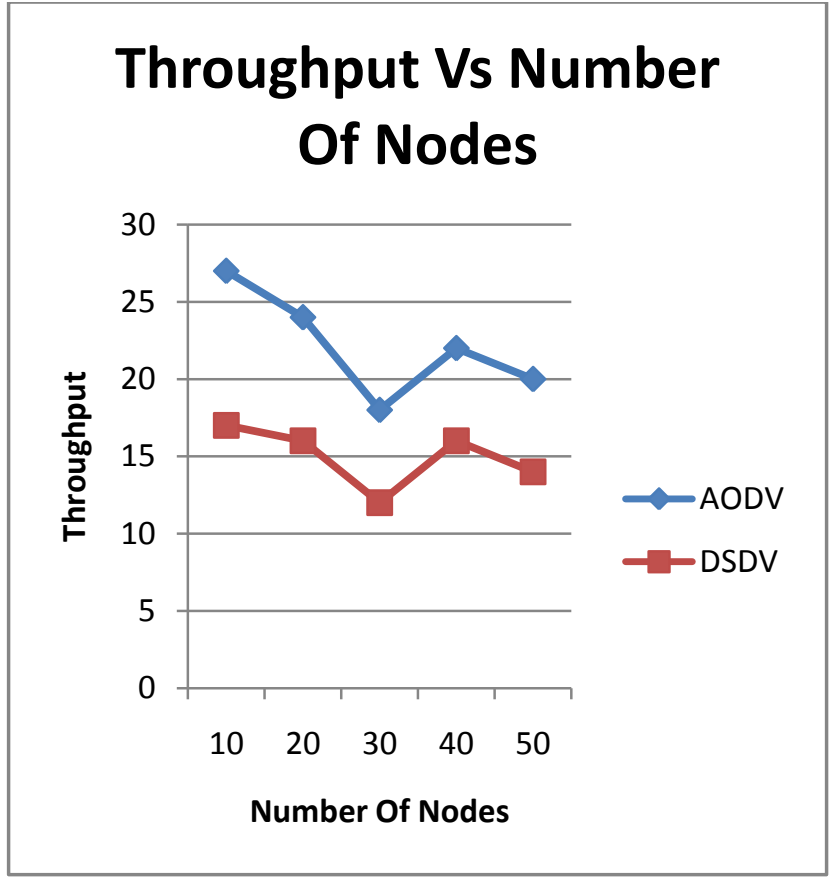

Fig 8. Throughput Analysis

There are some other parameter which are improved while following our proposed system[10][11].

\subsection{Analysis:}

Here before analyzing System we assume that $10 \%$ of vehicle are VIP or emergency vehicles and Speed of V1=44 and $\mathrm{V} 3=60$ and $\mathrm{D}=1000 \mathrm{~m}$ and $\mathrm{D} 1=500 \mathrm{~m} \mathrm{t} 2=5 \mathrm{sec}$ and $\mathrm{t} 3=2 \mathrm{sec}$.

\subsubsection{Air Pollution:-}

So Air Pollution for Existing System $=\{(D 1 / V 1) * n * E p+(D-$ D1)/V1*n*Ep\}

Air Pollution for Proposed System $=$

$\{(\mathrm{D} 1 / \mathrm{V} 1) * \mathrm{n} * \mathrm{Ep}+(\mathrm{D}-\mathrm{D} 1) / \mathrm{V} 3 *(10 \%$ of $\mathrm{n}+[\{\mathrm{n}-10 \%$ of n\}/2]*Ep\}.

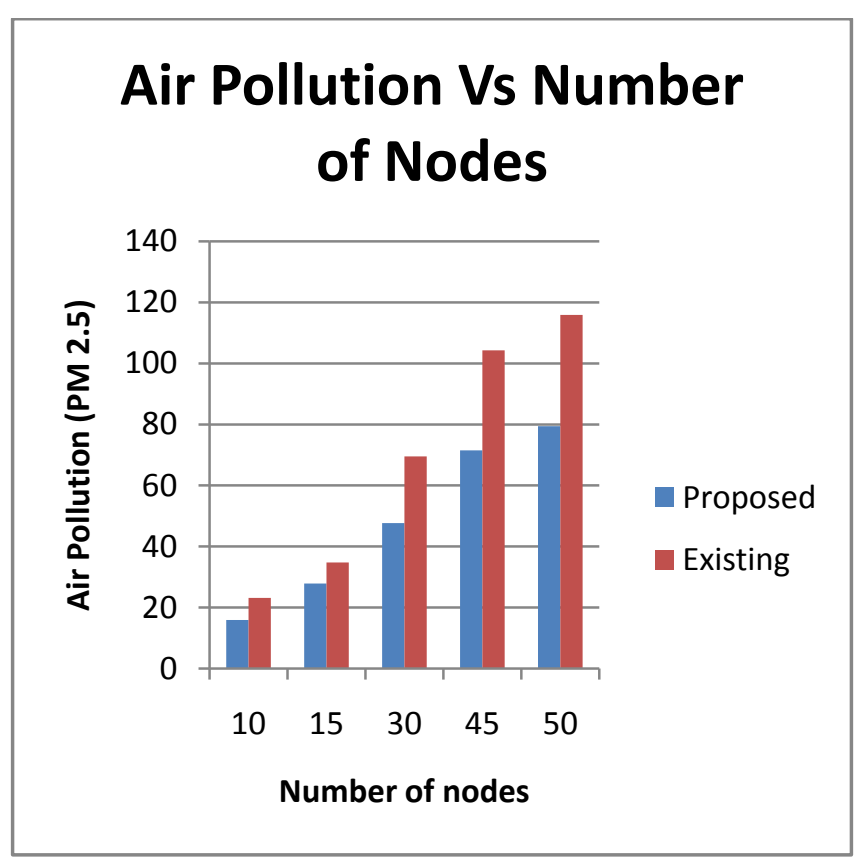

Fig 9. Air Pollution Vs Number Of Nodes 
4.4.2 Time Taken To Reach Destination:-

Time taken by $\mathrm{n}$ vehicle to reach destination in Existing System.

Texis $=\mathrm{n} *[(\mathrm{D} 1 / \mathrm{V} 1)+\mathrm{t} 2+(\mathrm{D}-\mathrm{D} 1) / \mathrm{V} 2]$.

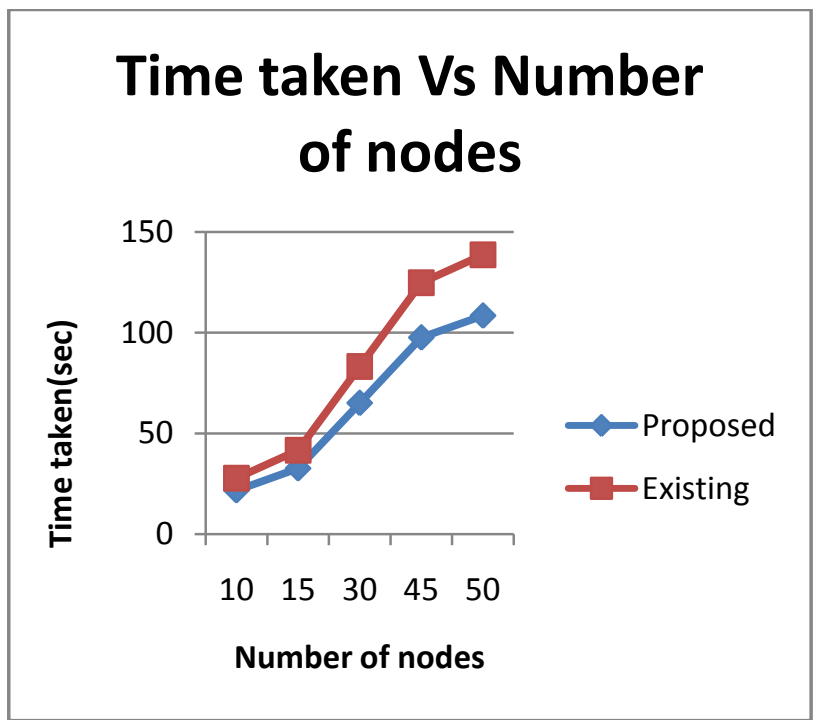

Fig 10. Time Taken To Reach Destiation Vs Number Of Nodes

Time taken by $\mathrm{n}$ vehicle to reach destination in Proposed System.

Tpro $=\mathrm{n}^{*}[(\mathrm{D} 1 / \mathrm{V} 1)+\mathrm{t} 3+(\mathrm{D}-\mathrm{D} 1) / \mathrm{V} 3]$.

4.4.3 Vehicle Density:-

Vexis $=$ Number of Lanes* Number of Nodes

Vpro $=$ Number of Lanes $*$ (Total Number of Vehicles Number of vehicle not allowed on particular day $+10 \%$ of total vehicle ( VIP vehicle) )

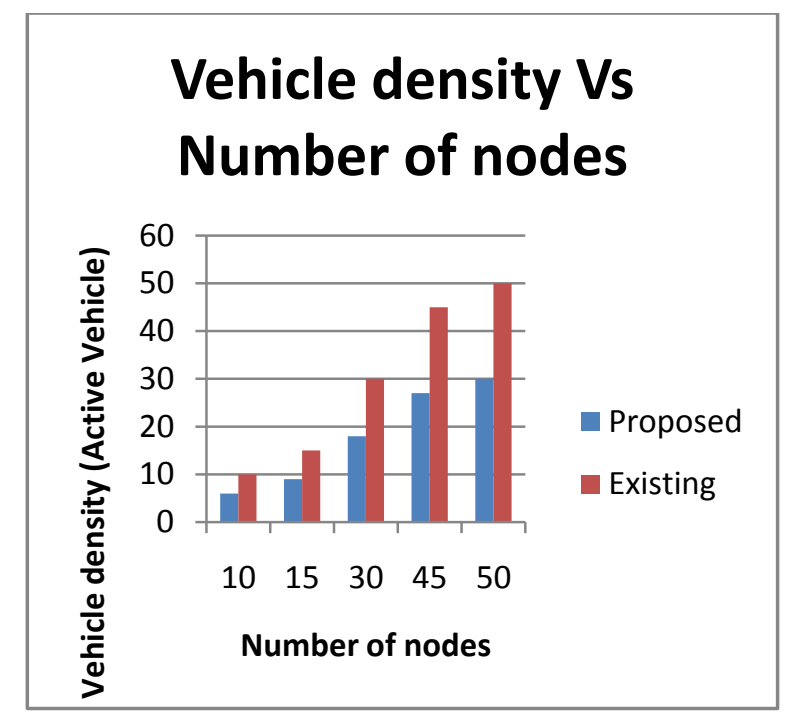

Fig. 11. Vehicle Density Vs Number Of Nodes

\subsubsection{Fuel Consumption}

This is one of another benefits of our proposed system. Since due to decrease vehicle density there is decrease in fuel consumption .

Fuel Consumption $=$ Vehicle Density $*$ Mean Fuel consumption of Vehicle.
In India Mean Fuel Consumption of Vehicle $=18 \mathrm{~km} /$ litres.

So to cover $1 \mathrm{~km}$ we have to get $=1 / 18=0.0555$ litres .

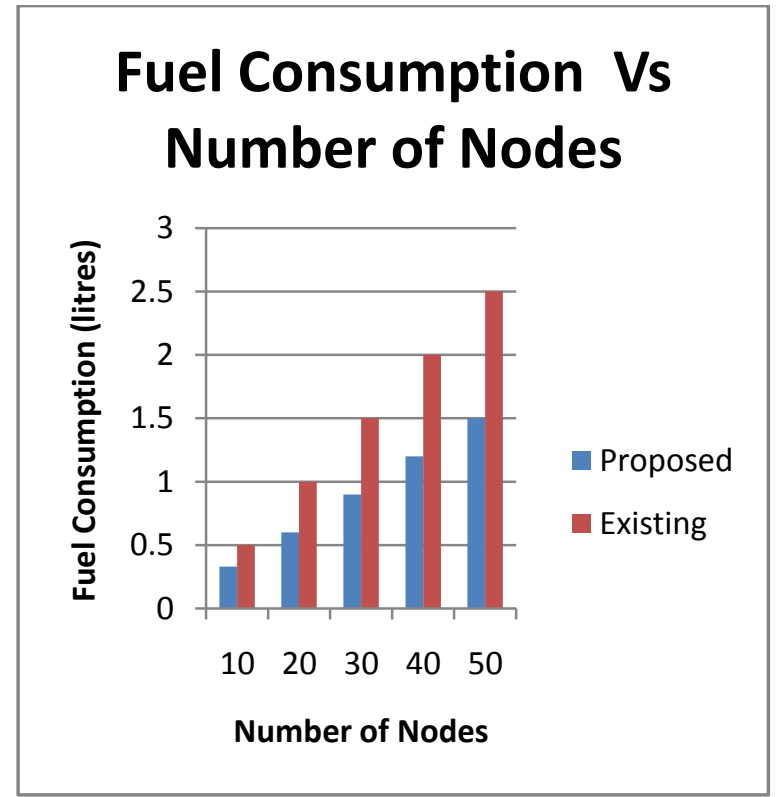

Fig 12. Fuel Consumption Vs Number Of Nodes

5. CONCLUSION AND FUTURE SCOPE In this paper, we first try to give introduction about the Vanet,its characterstics and its application .In second Section we try to elaborate how our existing system works. In ths section we also cover what are the drawback of our existing system.then we elaborate our proposed System to overcome the problem that arises in our existing System.Our proposed system work on odd-even policy for better handling and managing of congestion in city. the main objective of our proposed System is that allow only odd vehicle to travel on odd day and vice-versa.

For Future scope we can add Continuous Internet Connectivity with efficient Handover for vehicle user for fast and reliable information.

6. REFERENCES

[1] "Vehicular Ad hoc Network (VANETs): A Review" Divya Chadha1, Reena2 Assistant Professor, MICT\&BM, Maharishi Markandeshwar University,Mullana, Ambala, Haryana, India1 M. Phil Scholar, M.M.I.C.T\&B.M, Maharishi Markandeshwa University, Mullana, Ambala, Haryana, India2 in IJERCCE vol. 3 Issue 3 , March 2015.

[2] "Enhancing VANET Connectivity Through Roadside Units on Highways" Sok-Ian Sou and Ozan K.Tonguz, Member, IEEE TRANSACTIONS ON VEHICULAR TECHNOLOGY, VOL. 60, NO. 8, OCTOBER 2011

[3] "A Secure and Efficient Communication in VANE" Debasis Giri and Durbadal Chattaraj Department of Computer Science and Engineering, Haldia Institute of Technology Haldia-721657, India in IEEE 2010.

[4] "Providing Ubiquitous Communication using Road-Side Units in VANET Systems: Unveiling the Challenges", Arindam Ghosh, Vishnu Vardhan, Glenford Mapp, Orhan Gemikonakli, Jonathan Loo Department of Computer and Communications Engineering Middlesex University London, United Kingdom. IN $13^{T H}$ 
INTERNATIONAL CONFERENCE ON ITS TECHNOLOGY

[5] "An Intelligent Secure Traffic Management System Based on Vanet" , Abinaya.E1, Sekar., Abinaya.E1, Sekar in IOSR Volume-9,Issue 1 Jan2010.

[6] "Way To Green : Intelligent Traffic Control With Vanet" Jini Kuriakose,Aromal C M,Gayathry K.V International Journal of Computer Engineering and Technology (IJCET) Volume 5 Issue 12, December 2014.

[7] Case Study : "Controlling millions of vehicles Beijing, China's Traffic policy package".

[8] Case Study on : "Vehicle Use Restriction and Limit."
[9] "Will a Driving Restriction Policy Reduce Car Tips? A Case Study of +-Beijing, China", Lanlan Wang, Jintao $\mathrm{Xu}$, Xinye Zheng and Ping Qin.

[10] "Air Pollution Due To Road Transportation In India: A Review On Assessment And Reduction Strategies" Shrivastava R. K., Saxena Neeta and Gautam Geeta Department of Mathematics, S.M.S. Govt. College, Gwalior (INDIA), Department of Mathematics , I.T.M. Gwalior (INDIA).

[11] "Air quality benefits of odd-even vehicle ationing programmein Delhi:" An analysis of week 1 Environment Pollution (Prevention and Control) Authority for NCR January 8, 2016. 